\title{
Mild encephalopathy with reversible lesion of the splenium caused by infectious endocarditis
}

\author{
Rainer Wunn, Christine M. Fellner, Franz A. Fellner \\ Institute of Radiology, AKH Linz, Austria \\ Correspondence: Franz A. Fellner, M.D. Head of the Institute of Radiology. Address: Krankenhausstr. 9, A-4020 Linz, \\ Austria. E-mail: franz.fellner@akh.linz.at \\ Received: November 14, 2013 \\ Accepted: December 11, 2013 \\ Online Published: December 16, 2013 \\ DOI : $10.5430 / j b g c . v 4 n 1 p 47$ \\ URL: http://dx.doi.org/10.5430/jbgc.v4n1p47
}

\begin{abstract}
Mild encephalopathy with reversible lesion of the splenium of corpus callosum is a rare disease revealing mild neurological symptoms, such as disturbance, ataxia, vertigo and in some cases headache. Magnetic resonance signal alterations in the splenium of the corpus callosum can be found in patients suffering from different diseases, mostly viral infections, but also seizures, antiepileptic drug therapy, bacterial infections, hypoglycaemia, Wernicke encephalopathy, Marchiafava Bignami disease, hemolytic uremic syndrome, acute urinary retention, and acute axonal trauma.

We report a 32-year old man admitted to our hospital with fever and vomiting. In the neurological examination he presented with mild dizziness and confusion. Until that time no cardiac symptoms had been reported. Due to the neurological symptoms an MR scan of the brain was performed visualizing an area of restricted diffusion with a diameter of $7 \mathrm{~mm}$ located in the central portion of the splenium of the corpus callosum being hyperintense on Fluid Attenuated Inversion Recovery (FLAIR) and T2-weighted turbo spin-echo images. Furthermore, multiple punctate lesions with similar appearance on diffusion weighted imaging were found in both hemispheres suspicious of embolic ischemic etiology. Consecutive transesophageal echocardiography revealed endocarditis of the mitral valve.

Transient lesions of the splenium recovering within a month are not specific and there is a wide range of differential diagnoses. They may indicate severe disease despite of initially mild neurological symptoms. Pathophysiology of these lesions is not clearly understood yet, even if there exist some - however not proven - theories, such as rapidly resolving intramyelinic edema or influx of inflammatory cells associated with cytotoxic edema.
\end{abstract}

\section{Key words}

Magnetic resonance, Reversible splenial lesion, Encephalopathy, Diffusion, Endocarditis

\section{I ntroduction}

In the Western world infectious endocarditis is a rare disease since the application of antibotic drugs. Patients with cardiac failure or scheduled for cardiac valve repair receive prophylactic antibiotic treatment in order to avoid valve infection or other infectious lesions. Untreated, the outcome of endocarditis is very poor and may implicate fatal complications. The most common location is the mitral valve developing valvular insufficiency, stenosis or a combination of both. A well-known complication is the occurrence of arterial septic emboli producing multiple ischemias and infectious foci all over the body. Another complication may be chorea minor as an autoimmune reaction after bacterial endocarditis. The 
symptoms of the latter should not be mistaken for the symptoms caused by Mild Encephalopathy with Reversible Lesion of the Splenium of the corpus callosum (MERS). MERS presents with a clear focus of MR signal alterations located in the splenium of the corpus callosum. However, this lesion is not specific. Otherwise, there may be associated brain lesions that may lead one to the correct diagnosis.

\section{Case presentation}

A 32-year old man without any disease history was admitted to our hospital presenting fever (up to $40^{\circ} \mathrm{C}$ ) and vomiting. Plain chest radiography was normal. Blood level leukocytes were elevated, as well as acute phase proteins (IL 6 and CRP). In the neurological examination dizziness and mild confusion were obvious. For this reason a cerebral MR scan was initiated and performed with the following standard imaging protocol on a $1.5 \mathrm{~T}$ scanner (Magnetom Avanto, Siemens, Erlangen, Germany) using a standard phased-array head coil: T2-weighted turbo spin-echo, turbo Fluid Attenuated Inversion Recovery (FLAIR), Diffusion-weighted imaging, T2*-weighted gradient-echo, and T1-weighted spin-echo before and after i.v. administration of $8 \mathrm{ml}$ Gadobutrol (Gadovist, Bayer Healthcare, Germany). Slice thickness in all sequences was $5 \mathrm{~mm}$ with a $10 \%$ gap. Finally, a 3D gradient-echo sequence (MP-RAGE) with a slice thickness of $1.0 \mathrm{~mm}$ was acquired in the sagittal plane (for multiplanar reconstructions).

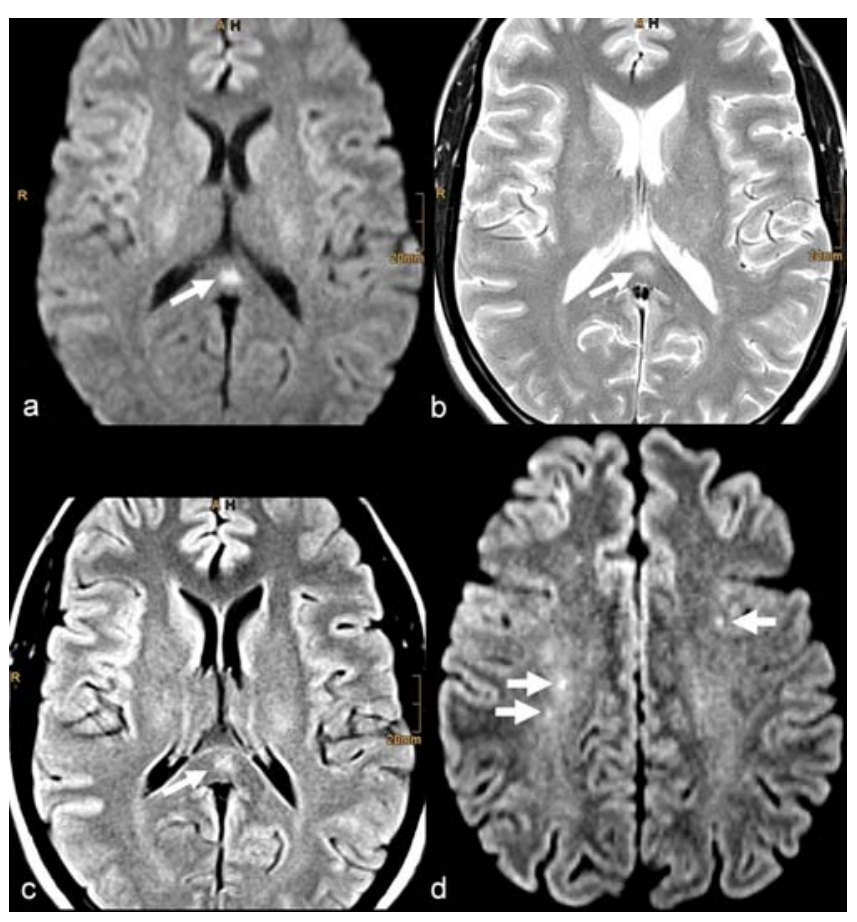

Figure 1. Diffusion-weighted imaging (a) shows an area with significant restriction of diffusion within the splenium of the corpus callosum. This lesion is slightly hyperintense on T2-weighted turbo spin-echo (b) and turbo FLAIR (c). Furthermore, multiple punctate lesions with diffusion restriction are found in both cerebral hemispheres suspicious of embolic ischemic lesions (d).

Image evaluation revealed a lesion with significant reduction of diffusion, located within the central portion of the splenium of the corpus callosum. This lesion was slightly hyperintense on FLAIR and T2-weighted turbo spin-echo images and did not enhance gadolinium. Furthermore, multiple punctate lesions were found in both cerebral hemispheres, also with reduction of diffusion, appearing as multiple embolic ischemic lesions (see Figure 1), also without gadolinium uptake. Subsequently, transesophageal echocardiography (TEE) beneath ultrasound of the cervical vessels was performed to find a possible source of the emboli. TEE revealed mitral valve endocarditis that had led to mitral insufficiency. Blood culture was positive for staphylococcus aureus. During cardiac surgery multiple bacterial vegetations were found with a diameter up to $7 \mathrm{~mm}$ associated with perforation of one of the leaflets that was treated with a pericardial patch. After surgery, the patient convalesced rapidly under antibiotic therapy. Concerning the neurological symptoms, the patient obtained complete recovery. Final blood tests were normal.

On day 11 after surgery a repeat MR scan was performed demonstrating full remission of the lesion in the dorsal 
corpus callosum. Furthermore, there was complete remission of the diffusion reduction of the multiple punctate lesions in both cerebral hemispheres, but now with significant gadolinium uptake according to the usual time course of ischemic lesions (see Figure 2). Another source of infection could not be found. The patient left the hospital without any symptoms.

Figure 2. Follow-up MR after treatment demonstrates complete remission of the splenial lesion on Diffusion-weighted imaging (a), T2-weighted turbo spin-echo (b), and turbo FLAIR (c). The multiple punctate lesions in both hemispheres reveal now significant gadolinium uptake (subacute stage of ischemia) on the contrast-enhanced T1-weighted sequence (d).

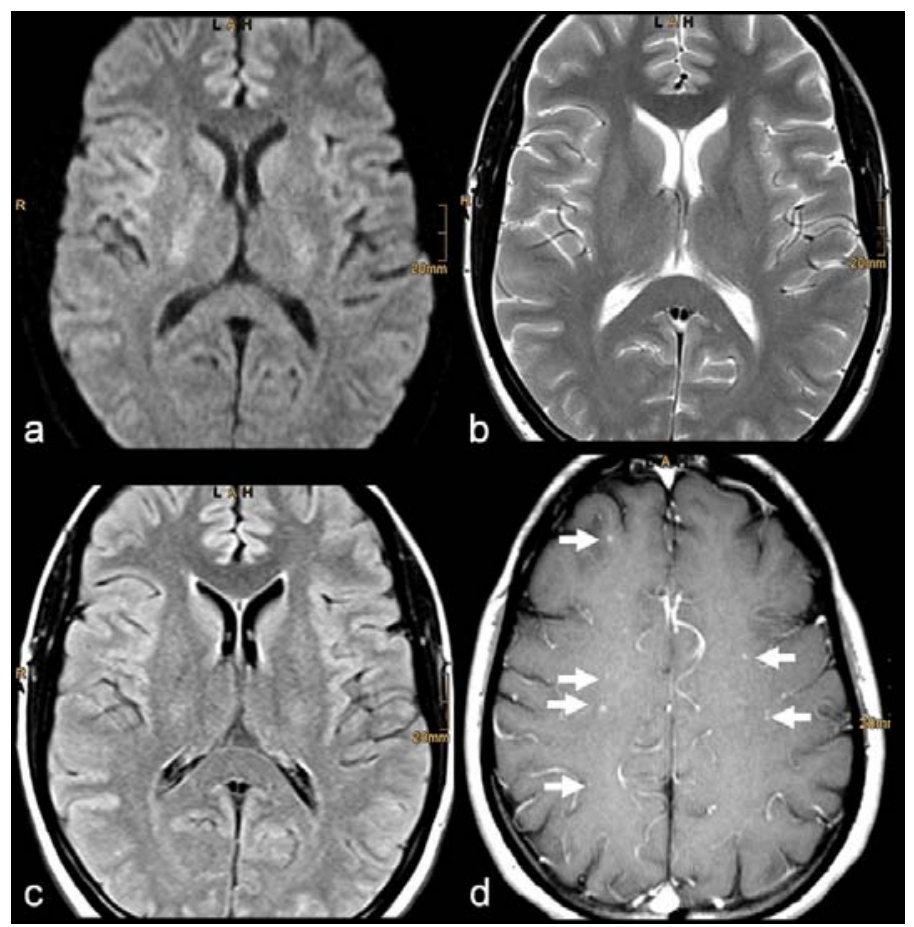

\section{Discussion}

MERS presents with a clear focus of MR signal alterations located in the splenium of the corpus callosum. However, this lesion is not specific. Otherwise, there may be associated brain lesions that may lead one to the correct diagnosis. Clinical symptoms are mild confusion, gait ataxia, and seizures. Usually, there is complete recovery of the symptoms and the splenial lesion in MRI ${ }^{[1]}$. Such a splenium lesion may occur in a lot of other conditions, such as seizures, antiepileptic drug therapy, viral infections - mostly influenza - bacterial infections, hypoglycaemia, Wernicke encephalopathy, Marchiafava Bignami disease, hemolytic uremic syndrome, acute urinary retention, and acute axonal trauma ${ }^{[2-5]}$. Recent reports describe involvement of the whole corpus callosum and even adjacent deep white matter, also in that cases with complete recovery within four weeks ${ }^{[6-9]}$. The pathophysiology of MERS is not really understood yet.

Indeed, the etiology and pathophysiology of transient splenial lesions, as shown in this case of MERS, are unclear yet. Tada et al. ${ }^{[1]}$ consider two theories concerning the lesion within the corpus callosum: The first theory assumes the lesion to arise secondary to an intramyelinic edema due to separation of the myelin layers.

The second theory discusses an influx of inflammatory cells and macromolecules in combination with cytotoxic edema. Furthermore, interstitial edema is considered also, because MERS has been reported even in neonates, in whom the corpus callosum is not myelinated yet ${ }^{[4]}$.

As mentioned, the main location of MERS is the dorsal corpus callosum, though recent papers describe involvement of other callosal parts and even the deep white matter. The preferred location in the posterior aspect of the corpus callosum is unclear yet, because a previous study evaluated that the callosal fibers do not vary ${ }^{[10]}$. Beyond that Takanashi et al. ${ }^{[11]}$ discuss direct viral invasion of the affected brain tissue. In addition or apart from the virus itself, unknown receptors or markers with special affinity to inflammatory cells or macromolecules, such as antibodies, could be responsible for the edema as in a cross reaction. Additionally of interest is the vascular supply. Kakou et al. ${ }^{[12]}$ demonstrated that the Published by Sciedu Press 
splenium is supplied by the terminal branches of the posterior cerebral artery, while the anterior aspects are fed by arterial branches from the internal carotid artery. In the sense of Olivot et al. ${ }^{[13]}$, the splenial alteration could be a reversible ischemic lesion.

MERS may arise in association with multiple other diseases, as mentioned above. Differential diagnosis must also include encephalomyelitis disseminata, ischemia, and lymphoma. Furthermore, another, clinically very important differential diagnosis is infectious endocarditis, as shown in this case report and previously described by Fukagawa $\mathrm{K}$ et al. ${ }^{[14]}$.

In conclusion, MERS - a rare entity - is an important red flag indicator for multiple other conditions, as shown in this case of infectious endocarditis, that have to be checked and ruled out carefully in each case.

\section{Conflicting interests}

The authors declare that they have no conflicting interests.

\section{Acknowledgement}

We express our thanks to Dr. Kaveh Akbari, for reviewing the manuscript, Prof. Dr. Gerhard Ransmayr (department of neurology), and Dr. Hannes Müller (department of heart \& vascular surgery) for providing us with the clinical data.

\section{References}

[1] Tada H, Takanashi J, Barkovich AJ, Oba H, Maeda M, Tsukahara H, et al. Clinically mild encephalitis/encephalopathy with a reversible splenial lesion. Neurology. 2004; 63: 1854-1858. PMid: 15557501. http://dx.doi.org/10.1212/01.WNL.0000144274.12174.CB

[2] Gallucci M, Limbucci N, Paonessa A, Caranci F. Reversible focal splenial lesions. Neuroradiology. 2007; 49: 541-544. PMid: 17522852. http://dx.doi.org/10.1007/s00234-007-0235-z

[3] Kitami M, Kubo S, Nakamura, S, Shiozawa S, Isobe H, Furukawa Y. Acute urinary retention in a 23-year-old woman with mild encephalopathy with a reversible splinial lesion: a case report. J Medical Case Reports. 2011; 5: 159. PMid: 21507219. http://dx.doi.org/10.1186/1752-1947-5-159

[4] Takanashi J, Hirasawa K, Tada H. Reversible restricted diffusion of entire corpus callosum. J Neurol Sci. 2006; 247: 101-104. PMid: 16631795. http://dx.doi.org/10.1016/j.jns.2006.03.015

[5] Bulakbasi N, Kocaoglu M, Tayfun C, Ucoz T. Transient splenial lesion of the corpus callosum in clinically mild influenza-associated encephalitis/encephalopathy. AJNR. 2006; 27: 1983-86. PMid: 17032879.

[6] Okumura A, Noda E, Ikuta T, Natsume J, Nagai T, Gotoh Y, et al. Transient encephalopathy with reversible white matter lesions in children. Neuroped. 2006; 37: 159-162. PMid: 16967368. http://dx.doi.org/10.1055/s-2006-924430

[7] Takanashi J, Barkovich AJ, Shiihara T, Tada H, Kawatani M, Tsukahara H, et al. Widening spectrum of a reversible splenial lesion with transiently reduced diffusion. AJNR. 2006; 27: 836-838. PMid: 16611774.

[8] Osuka S, Imai H, Ishikawa E, Matsushita A, Yamamoto T, Nozue H, et al. Mild encephalitis/encephalopathy with a reversible splenial lesion: Evaluation by diffusion tensor imaging - Two case reports. Neurol Med Chir (Tokyo). 2010; 50: 1118-1122. http://dx.doi.org/10.2176/nmc.50.1118

[9] Takahashi J, Barkovich AJ, Yamaguchi K, et al. Influenza-associated encephalitis/encephalopathy with a rerversible lesion in the splenium of the corpus callosum: a case report and literature review. AJNR. 2004; 25: 798-802.

[10] Aboitiz F, Scheibel AB, Fisher RS, Zaidel E. Fiber composition of the human corpus callosum. Brain Res. 1992; 598: $143-153$. http://dx.doi.org/10.1016/0006-8993(92)90178-C

[11] Kobata R, Tsukahara H, Nakai A, Tanizawa A, Ishimori Y, Kawamura Y, et al. Transient MR signal changes in the splenium of the corpus callosum in rotavirus encephalopathy: value of diffusion-weighted imaging. J Comput Assist Tomogr. 2002; 26: 825-828. PMid: 12439323. http://dx.doi.org/10.1097/00004728-200209000-00028

[12] Kakou M, Velut S, Destrieux C. Vascularisation artérielle et veineuse du corps calleux. Neurochir. 1998; 4: 31-37.

[13] Olivot JM, Mlynash M, Thijs VN, Purushotham A, Kemp S, Lansberg MG. et al. Relationships between cerebral perfusion and reversibility of acute diffusion lesions in DEFUSE. Insights from RADAR. Stroke. 2009; 40: 1692-1697. PMid: 19299632. http://dx.doi.org/10.1161/STROKEAHA.108.538082

[14] Fukagawa K, Izumi M, Higuchi K, Adachi Y. Reversible splenial lesion associated with Staphylococcus aureus endocarditis. Intern Med. 2013; 52: 1147-1148. PMid: 23676609. http://dx.doi.org/10.2169/internalmedicine.52.9286 